\title{
The relationship between cognitive and physical function among residents of a Czech senior home
}

\author{
Annelies Matthé ${ }^{1}$, Donald N. Roberson Jr. ${ }^{2, *}$, and Yael Netz ${ }^{3}$ \\ ${ }^{\text {I}}$ Special Olympics Belgium, Brussels, Belgium; ${ }^{2}$ Faculty of Physical Culture, Palacký University Olomouc, Olomouc, Czech \\ Republic; and ${ }^{3}$ Wingate College, Wingate Institute, Netanya, Israel
}

Copyright: (C) 2015 A. Matthé et al. This is an open access article licensed under the Creative Commons Attribution License (http://creativecommons.org/licenses/by/4.0/).

\begin{abstract}
Background: The decline in cognition and physical fitness is common in advanced age. Objective: The relationship between cognition and aerobic capacity was compared to the relationship between cognition and balance. Methods: Twenty one females and six male residents of a Czech senior center participated in the study (mean age: $77.5 \pm 7.0$; range: $62-86$ years). The Mini Mental State Examination (MMSE) was used for assessing cognition, the Berg Balance Scale (BBS) for assessing balance, and the 6-Minute Walk Test (6MWT) assessed physical fitness. Based on the MMSE scores, two groups of cognitive functioning were formed - high and low. Results: Participants in the "high MMSE" group reached a significantly higher score on the 6MWT $(p=.01)$ than the "low MMSE" group. Group differences on the BBS were marginally significant $(p=.07, d=0.6)$. Conclusions: Based on this sample, the level of physical fitness can be explained by cognitive functioning, while that of balance should be further studied in its relationship to cognitive functioning.
\end{abstract}

Keywords: aerobic fitness, balance, mental functioning, senior home

\section{Introduction}

Global demographics have changed in the last century, with the elderly population expanding and thereby becoming one of the most significant age groups. Figures from the World Health Organization (WHO) showed that by the year 2050 there will be up to 2 billion people aged 60 years or older, compared to 600 million people in 2000 (WHO, 2011). These older people often face health problems including physical (American College of Sports Medicine - ACSM, 2009) and cognitive deteriorations (Park, O'Connell, \& Thomson, 2003). As they age, they perform less physical activity which is also less intense (ACSM, 2009). Together with the natural effects of aging this induces, among others, problems with their equilibrium (Konrad, Girardi, \& Helfert, 1999) and a decrease of their maximum aerobic capacity and skeletal muscle performance (ACSM, 2009). The cognitive problems are very diverse as they depend on the cognitive domain(s)

\footnotetext{
* Address for correspondence: Donald N. Roberson Jr., Department of Social Sciences in Kinanthropology, Faculty of Physical Culture, Palacký University Olomouc, třída Míru 117, 77111 Olomouc, Czech Republic. E-mail: donald.roberson@upol.cz
}

where the degeneration is located (Park et al., 2003). They can be further characterized by the presence of amnesia (Park et al., 2003). Both declines are independently associated with high mortality rates (Kokkinos et al., 2010; van Gelder, Tijhuis, Kalmijn, Giampaoli, \& Kromhout, 2007) and result in high social-economic costs for every national health care system (Kokkinos et al., 2010; Park et al., 2003).

Previous research showed an association between cognitive function and different measures of physical performance in an elderly population: aerobic fitness (Baldasseroni et al., 2009; Netz, Dwolatzky, Zinker, Argov, \& Agmon, 2011), balance (Rolland et al., 2009; Voos, Custódio, \& Malaguias, 2011), and gait speed (Atkinson et al., 2010; Atkinson et al., 2007; Nieto, Albert, Morrow, \& Saxton, 2008). A meta-analysis of Smith et al. (2010) further confirmed the relationship between aerobic exercise training and cognition. Twenty two randomized control trials were included in this study. They all observed the effects on cognition after an exercise program that included aerobic exercises. While the association between cognitive function and aerobic performance has been well studied, the association with balance is investigated less. The results of the study by Brown, Lui-Ambrose, Tate, 
and Lord (2009), Voos et al. (2011), and Rolland et al. (2009) suggest that the participants improved their cognitive function after a training program consisting of resistance and balance training. Balance as well as fear of falling is one of the most important concerns for older adults. This important consideration showed that often the overlooked issue of balance should be further studied.

This unique association of cognition and aerobic fitness with the association of cognition and balance is rarely studied. Therefore the objective of this research was to explore to which dimension of physical function - balance or aerobic fitness - cognitive function is more related. Balance is overlooked in relationships with other research topics, and balance exercises are often placed behind the more popular topics of aerobics or exercise classes. Two hypotheses were assumed: 1) there is a relationship between cognition and aerobic fitness; 2) there is a relationship between cognition and balance.

\section{Methods}

\section{Participants}

The participants were residents of a senior home in the Czech Republic. Residents were included if they were older than 60 years, if they were able to tolerate standing or walking for six minutes without shortness of breath, chcest pain or joint pain, and if they had a score of $\geq 20 / 30$ on the Mini Mental State Examination. Residents were excluded if they had a severe chronic disease, demonstrated signs of delirium and/or if they were not able to walk as a result of an acute illness, motor disability or visual defect. The use of crutches was allowed. Because the lead researcher was not Czech, we incorporated the use of translators who were also trained in how to use the tests. We were careful to fit in with the social situation within the environment of the senior home, and we followed the suggestions of the manager of the center.

The cutoff point of 20/30 for the MMSE is based on the finding by Folstein, Folstein, and McHugh (1975), Logsdon, Gibbons, McCurry, and Teri (2002) and Feinberg and Whitlatch (2001). Folstein et al. (1975) stated that dementia starts with scores of 20/30 or less. The study by Logdson et al. (2002) showed that people with an MMSE greater than ten are able to assess their quality of life on a reliable and valid way, and the participants in the study by Feinberg and Whitlatch (2001) with an MMSE greater than 13, responded reliably on questions about demographics, choices, preferences, and daily living. Based on these findings, it can be assumed that the residents were able to understand the test instructions and reliably answered the questions of the initial assessment. In total, twenty seven residents met these criteria. They all gave a written informed consent to participate. The study was approved by the Ethic Committee of the Faculty of Physical Culture, Palacký University Olomouc.

\section{Cognitive assessment}

The MMSE was used to assess global cognitive function (Folstein et al., 1975). The test was originally developed by Folstein et al. (1975) as a screening tool for cognitive impairment in neuro-geriatric patients. However, it has been used as an outcome measure in studies assessing relationships between physical activity (Muscari et al., 2010) or physical fitness (Baldasseroni et al., 2010) and cognitive function. Different cognitive functions are evaluated ranging from orientation in time and space, attention, recall and ability to name (Folstein et al., 1975). A maximum score of 30 can be obtained, with higher scores indicating a better cognitive function.

\section{Assessment of aerobic fitness}

The Six-Minute Walk Test (6MWT) was used to assess physical fitness (Butland, Pang, Gross, Woodcock, \& Geddes, 1982). According to Kervio, Carre, and Ville (2003), who tested healthy elderly adults, this is a submaximal exercise test at almost $80 \%$ of $\mathrm{VO}_{2}$ max. Participants are instructed to walk as much distance as possible, at a self-selected pace, in six minutes. The test was performed in a quiet corridor and a walking course of $30 \mathrm{~m}$ in length was measured according to the American Thoracic Society (2002). A chair was placed at each end of the $30 \mathrm{~m}$ walk path in case the participants needed to rest. Standard verbal encouragement was given every minute. If participants used crutches in their daily activities, they were allowed to use them during the test.

\section{Assessment of balance}

Balance was tested using the Berg Balance Scale (BBS) (Berg, Wood-Dauphinee, Williams, \& Maki, 1992). The test is comprised of fourteen tasks, ranging from static to dynamic exercises. With each task a maximum score of four can be earned, a higher score is appointed when the person performs the exercise better. The maximum score that can be reached on the test is 56 . A higher score corresponds with a better balance (Holbein-Jenny, Billek-Sawhney, Beckman, \& Smith, 2005; Steffen, Hacker, \& Mollinger, 2002).

\section{Procedure}

All residents were invited to an information meeting in which the study was explained. Since only three 
residents attended the meeting, another strategy was adopted. All eligible residents (as defined by the staff of the senior home) were personally invited by an employee of the retirement center who was briefed on the purpose, content and procedure of the study. Residents who were willing to participate were assigned to a certain test date and hour. An instruction note containing basic information and instructions, such as test location and the instruction to wear light clothing was provided to each participant. All tests were performed in the same setting.

A short interview with each participant was conducted, followed by the cognitive assessment, balance assessment and aerobic fitness assessment. During the interview the following information was collected: date of birth, gender, education level, smoking habits and feelings of depression (self-diagnosed). Furthermore, the date and time of the test were noted on the test chart.

The interview, cognitive assessment, and balance assessment were assessed in a private room. The assessment of aerobic fitness was performed in the corridor next to that room. Two translators were used to insure accuracy of information and discussion. They were familiar with the tests, helped with all translations, and scored the initial and cognitive assessments. If patients stopped during the 6MWT it was noted on the test chart.

\section{Data analysis}

The demographic and clinical characteristics were expressed as mean \pm standard deviation. These characteristics were calculated for the following groups - the entire study sample, female, male, participants who used a walking aid, participants who did not use a walking aid, participants who were tested before noon and participants who were tested in the afternoon. The Mann-Whitney $U$ test was used to calculate the differences between the following subgroups - female/male, walking aid/no walking aid, before noon/after noon. Further comparisons were made for participants who did and did not stop during the 6MWT, for smokers and non-smokers, and for participants with and without self-diagnosed depression. Finally, the study sample was divided into two groups: low MMSE score $(<27)$ and high MMSE score ( $\geq 27)$.

A cut off score of 27 was chosen based on previous studies (Dik et al., 2000; Poynter, Kwan, Sayer, \& Vassallo, 2011). The differences among these two groups were also analyzed using the Mann-Whitney $U$ test. For all comparisons, Cohen's $d$ effect size was used to evaluate "the standardized difference between means" for all of the above listed subgroup comparisons (McCartney \& Rosenthal, 2000, p. 174). The following convention was adopted to evaluate the size of the effect: Cohen's $d$ effect size of 0.20 was considered small, 0.50 moderate, and 0.80 large (McCartney \& Rosenthal, 2000). Spearman's correlation was applied to evaluate the association of each variable with each other. A significance level of $p=.05$ was used in all statistical analyses. The statistical analysis was conducted using STATISTICA (Version 9; StatSoft, Tulsa, OK, USA).

\section{Results}

The demographic characteristics and the outcome parameters are presented for twenty seven residents, 21 females and 6 males, participating in the study (Table 1).

An additional comparison between participants who performed the test before noon and those who performed the test in the afternoon was performed to evaluate whether timing of the tests had an impact on the results. Although the men reached higher scores for all parameters, a significant difference was found only for education $(p=.01)$ and 6MWT $(p=.02)$. In addition, while gender comparison for BBS did not reach significance $(p=.06)$, Cohen's $d$ effect size was moderate $(d=0.7)$.

Significant differences were also observed between participants using a walking aid and participants who do not use a walking aid for the BBS $(p<.001)$ and the 6MWT $(p<.001)$. Participants using no walking aid

Table 1

Demographic characteristics of the sample $(M \pm S D)$

\begin{tabular}{lccc}
\hline & Whole group $(N=27)$ & Female $(n=21)$ & Male $(n=6)$ \\
\hline Age (years) & $77.5 \pm 7.0$ & $76.8 \pm 7.7$ & $80.0 \pm 3.3$ \\
Education (years) & $11.2 \pm 2.5$ & $10.6 \pm 2.3$ & $13.3 \pm 2.0$ \\
MMSE (score) & $26.0 \pm 2.9$ & $25.6 \pm 3.0$ & $27.2 \pm 2.3$ \\
BBS (score) & $47.3 \pm 7.8$ & $46.1 \pm 7.9$ & $51.7 \pm 5.9$ \\
6MWT (m) & $321.0 \pm 112.2$ & $298.5 \pm 104.0$ & $399.8 \pm 112.1$ \\
\hline
\end{tabular}

Note. $\quad$ MMSE $=$ Mini Mental State Examination, $\mathrm{BBS}=$ Berg Balance Scale, $6 \mathrm{MWT}=$ Six-Minute Walk Test. 
obtained higher scores on both tests. Higher MMSE scores were observed for participants using no walking aid, but these differences were not significant $(p=.08)$. Nevertheless, Cohen's $d$ effect size MMSE was high $(d=0.8)$.

Higher scores for all parameters were obtained by participants performing the tests in the afternoon, but differences were only significant for 6MWT $(p=.03)$. Although there was no significant difference for MMSE $(p=.01)$, a moderate to high Cohen's $d$ effect size $(d=0.7)$ was observed for MMSE.

Results for a low MMSE and high MMSE score are reported in Table 2. A trend for higher scores on BBS and 6MWT for participants with a high MMSE score was noted. The Mann-Whitney $U$ test demonstrated only a significant difference on the 6MWT $(p=.01)$ and marginally significant for the $\operatorname{BBS}(p=.07)$. A moderate effect size of $d=0.6$ was observed for the BBS.

A Spearman's correlation matrix was used for measuring relationships between variables (Table 3). Significant correlations were observed between MMSE and BBS $(p=.03)$, MMSE and 6MWT $(p<.01)$, BBS and 6MWT $(p<.01)$ and gender and 6MWT $(p=.01)$. Both "stopped at 6MWT" and "aid at 6MWT" correlated significantly with the score on $\operatorname{BBS}(p<.01$ and $p=.01$ respectively) and on $6 \mathrm{MWT}(p<.01$ and $p=.05$ respectively).

\section{Discussion}

The objective of this study was to compare the relationship between cognition and aerobic fitness to the relationship between cognition and balance utilizing a sample of older adults who are living in a senior center.

The first hypothesis was confirmed, since participants with a high level of cognition demonstrated a high level of physical fitness. These findings are supported by Baldasseroni et al. (2009), who investigated the relationship between cognition and aerobic capacity in a sample of older outpatients with chronic heart failure. The participants in our study were slightly older, more educated and reached higher mean distances. Since our study findings are similar to the findings of Baldasseroni et al. (2009), this may indicate that this relationship is typical for different groups of older individuals such as those in senior home residents and outpatients.

Atkinson et al. (2007) and Nieto et al. (2008) also showed a similar relationship between cognition (executive functioning) and gait speed. As gait speed determines the total distance walked in six minutes, the findings from Atkinson et al. (2007) and Nieto et al. (2008) support our findings.

A marginally significant relationship was found between cognitive function and balance. Since there was a non-significant relationship, the second hypothesis could not be confirmed. Contrary to the present study, a study by Li et al. (2010) showed that after a cognitive intervention, there was a training benefit in the single (one-leg) and double (two-legs) support standing balance. This different finding can be explained by a difference in the sample, with healthy community dwelling subjects in the study of Li et al. (2010) and residents of a senior home in our study. It is likely that the overall health condition of our participants was more deteriorated, with a higher chance of balance problems. Further, Li et al. (2010) only measured single and double standing balance support, which are only two of the 14 tasks that are included in the BBS. Voos et al. (2011) also found that participants with high levels of executive function also performed highly on the balance assessment. It should be kept in mind that our study showed a marginally significant relationship and that with a larger sample size, a significant relationship would have been possible. Since studies looking into this field are scarce, this study was an attempt to bridge this gap and to serve as a foundation for further research.

To the author's knowledge this is one of the first studies that compared these two physical measures and their relationship with cognitive function. It is likely

Table 2

Demographic and clinical characteristics $(M \pm S D)$ for the participants with low (score 20-26) and high (score 27-30) MMSE

\begin{tabular}{lcccc}
\hline & Low MMSE $(n=14)$ & High MMSE $(n=13)$ & $p^{\text {a }}$ & Cohen's $d$ \\
\hline Age (years) & $79.57 \pm 5.84$ & $75.31 \pm 7.74$ & .17 & 0.6 \\
Education (years) & $10.36 \pm 1.69$ & $12.08 \pm 2.99$ & .11 & 0.7 \\
BBS (score) & $45.00 \pm 8.10$ & $49.77 \pm 6.92$ & .07 & 0.6 \\
6MWT (m) & $264.29 \pm 118.94$ & $382.08 \pm 64.26$ & .01 & 1.0 \\
\hline
\end{tabular}

Note. $\quad$ MMSE $=$ Mini Mental State Examination, BBS $=$ Berg Balance Scale, $6 \mathrm{MWT}=$ Six-Minute Walk Test. ${ }^{\text {a} M a n n-W h i t n e y ~} U$ test. 


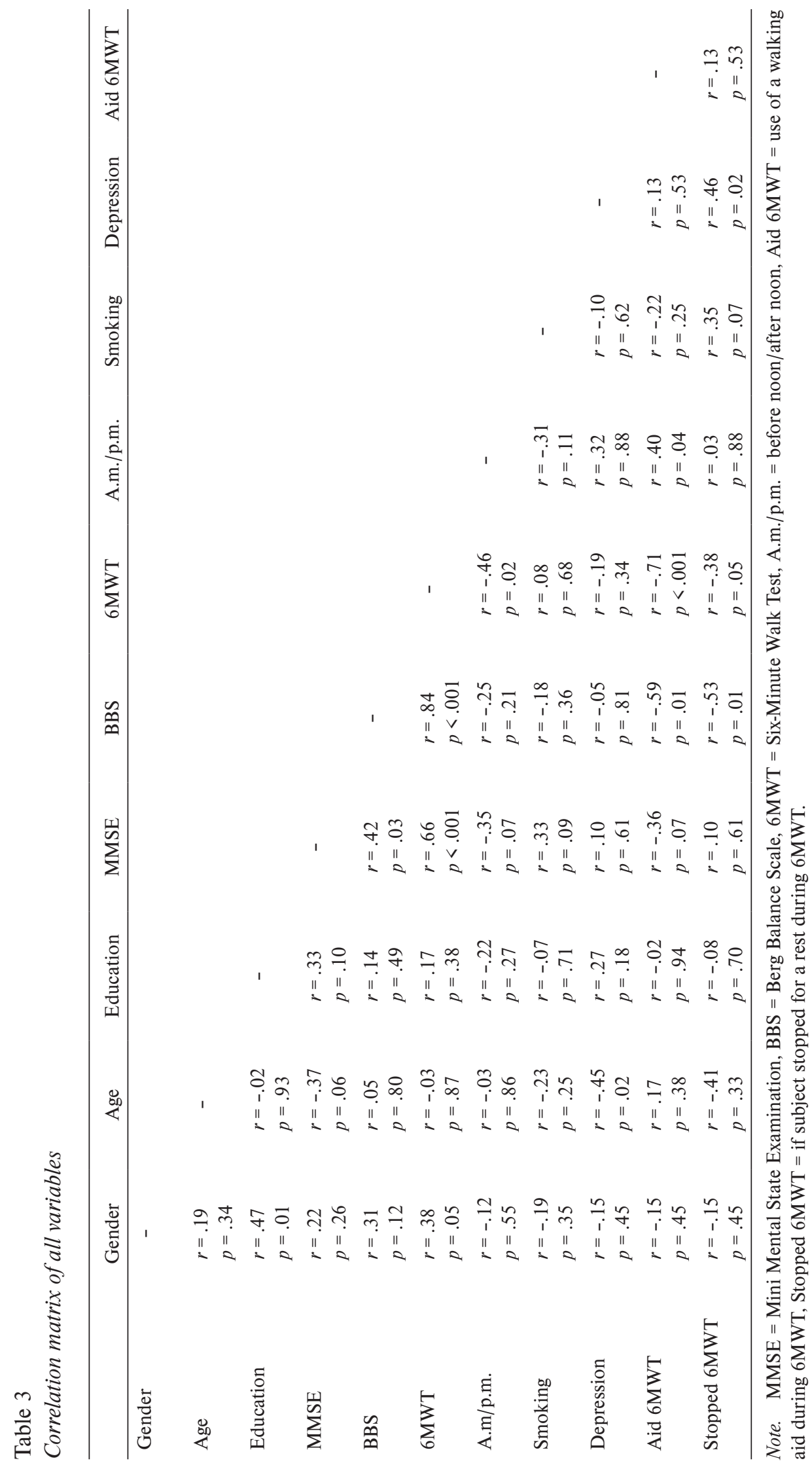


that for a high level of balance function, the visual, vestibular and proprioceptive systems (Konrad et al., 1999) are more important than a high level of cognitive function. As stated earlier, with a larger sample size, the marginally significant relationship between cognition and balance could become significant.

The results of this study showed that a different relationship exists between cognition and aerobic fitness and between cognition and balance. This implies a different underlying mechanism. Brown et al. (2009) stated that the condition of the cardiovascular system links cognition with aerobic fitness. In their study they showed that aerobic training improved or maintained the cognitive function through, among other issues, an increased vascularization. According to Rolland et al. (2009) the underlying mechanism between cognition and balance is less clear; it is possible that dysfunction in a lobe (parietal lobe) has both an effect on cognition as on balance (via a deficit in the visuospatial orientation). Depending on the type of exercise, different changes might be observed in the body and therefore have a different effect on the cognitive function of the elderly. Based on our study results, it is better to prescribe aerobic exercise for the elderly, in the context of cognitive training.

There is a need for randomized control trials as these studies can determine the cause-effect relationship, and therefore can identify the direction of the relationship between cognitive and physical function. Further studies are necessary to find out the mediating mechanisms of the relationship. Knowing the underlying mechanisms can lead to a better understanding of these relationships and can give caregivers new insights/ideas when preparing therapy for the elderly. Since our results showed that a stronger association exists between cognition and aerobic capacity compared to cognition and balance, it becomes even more important to know the underlying mechanisms in order to understand the difference in the two relationships. Future studies should increase their sample size in order to have large subgroups and to control for variables such as gender, education, and depression.

With 27 participants in our sample, there are several consequences and limitations for our research. First of all this low sample size does not allow us to generalize these results to the broader elderly population. We tried to overcome this disadvantage by including almost all residents of the retirement center that met the inclusion criteria. Further, as a result of the low sample size, we could not conduct statistical procedures analyzing the relationship between cognitive status and aerobic (or balance) fitness while controlling for moderating variables such as gender, education, or time of the test. Two other limitations of this research might be a volunteer bias and the two different translators that were assigned to score the different tests.

\section{Conclusion}

This study investigated the relationship between cognitive function and physical function. The findings of our study showed that the variance of physical fitness can be explained by cognitive function, whereas explaining the variance of balance by cognitive function is questionable. In terms of balance, this research adds to the current and scarce knowledge on the relationship between cognition and balance. The study is one of the few that has compared cognition and its relationship to different physical measures and that incorporated a sample from a retirement home.

\section{References}

American College of Sports Medicine (ACSM). (2009). Exercise and physical activity for older adults. Medicine \& Science in Sports \& Exercise, 41, 1510-1530.

American Thoracic Society. (2002). ATS statement: Guidelines for the six-minute walk test. American Journal of Respiratory and Critical Care Medicine, 166, 111-117.

Atkinson, H. H., Rapp, S. R., Williamson, J. D., Lovato, J., Absher, J. R., Gass, M., ... Espeland, M. A. (2010). The relationship between cognitive function and physical performance in older women: Results from the Women's Health Initiative Memory Study. Journal of Gerontology: Series A: Biological Sciences Medical Sciences, 65, 300-306.

Atkinson, H. H., Rosano, C., Simonsick, E. M., Williamson, J. D., Davis, C., Ambrosius, W. T., ... Health ABS Study. (2007). Cognitive function, gait speed decline, and comorbidities: The health, aging and body composition study. Journal of Gerontology: Series A: Biological Sciences Medical Sciences, 62, 844-850.

Baldasseroni, S., Mossello, E., Romboli, B., Orso, F., Colombi, C., Fumagalli, S., ... Marchionni N. (2010). Relationship between cognitive function and 6-minute walking test in older outpatients with chronic heart failure. Aging Clinical Experience Research, 22, 308-313.

Berg, K. O., Wood-Dauphinee, S. L., Williams, J. I., \& Maki, B. (1992). Measuring balance in the elderly: Validation of an instrument. Canadian Journal of Public Health, 83(Suppl. 2), S7-S11.

Butland, R. J., Pang, J., Gross, E. R., Woodcock, A. A., \& Geddes, D. M. (1982). Two, six, and 12 minute walking tests in respiratory disease. British Medical Journal (Clinical Research Ed.), 284, 1607-1608.

Brown, A. K., Lui-Ambrose, T., Tate, R., \& Lord, S. R. (2009). The effect of group-based exercise on cognitive performance and mood in seniors residing in intermediate care and self-care retirement facilities: A randomised controlled trial. British Journal of Sports Medicine, 43, 608-614. 
Dik, M. G., Jonker, C., Bouter, L. M., Geerlings, M. I., van Kamp, G. J., \& Deeg, D. J. (2000). APOE-epsilon4 is associated with memory decline in cognitively impaired elderly. Neurology, 54, 1492-1497.

Feinberg, L. F., \& Whitlatch, C. J. (2001). Are persons with cognitive impairment able to state consistent choices? Gerontologist, 41, 374-382.

Folstein, M. F., Folstein, S. E., \& McHugh, P. R. (1975). "Mini-mental state". A practical method for grading the cognitive state of patients for the clinician. Journal of Psychiatric Research, 12, 189-198.

Holbein-Jenny, M. A., Billek-Sawhney, B., Beckham, E., \& Smith, T. (2005). Balance in personal care home residents: A comparison of the Berg Balance Scale, the Multi-Directional Reach Test, and the Activities-Specific Balance Confidence Scale. Journal of Geriatric Physical Therapy, 28, 48-53.

Kervio, G., Carre, F., \& Ville, N. S. (2003). Reliability and intensity of the six-minute walk test in healthy elderly subjects. Medicine \& Science in Sports \& Exercise, 35, 169-174.

Kokkinos, P., Myers, J., Faselis, C., Panagiotakos, D. B., Doumas, M., Pittaras, A., Manolis, A., ... Fletcher, R. (2010). Exercise capacity and mortality in older men: A 20 year follow up study. Circulation, 122, 790-797.

Konrad, H. R., Girardi, M., \& Helfter, R. (1999). Balance and aging. Laryngoscope, 109, 1454-1460.

Li, K. Z. H., Roudaia, E., Lussie, M., Bherer, L., Leroux, A., \& McKinley, P. A. (2010). Benefits of cognitive dual task training on balance performance in healthy older adults. Journal of Gerontology: Series A: Biological Sciences \& Medical Sciences, 65, 1344-1352.

Logsdon, R. G., Gibbons, L. E., McCurry, S. M., \& Teri, L. (2002). Assessing quality of life in older adults with cognitive impairment. Psychosomatic Medicine, 64, 510-519.

McCartney, K., \& Rosenthal, R. (2000). Effect size, practical importance, and social policy for children. Child Development, 71, 173-180.

Muscari, A., Giannoni, C., Pierpaoli, L., Berzigotti, A., Maietta, P., Foschi, E., ... Zoli, M. (2010). Chronic endurance exercise training prevents aging-related cognitive decline in healthy older adults: A randomized controlled trial. International Journal of Geriatric Psychiatry, 25, 1055-1064.
Netz, Y., Dwolatzky, T., Zinker, Y., Argov, E., \& Agmon, R. (2011). Aerobic fitness and multidomain cognitive function in advanced age. International Psychogeriatrics, 23, 114-124.

Nieto, M. L., Albert, S. M., Morrow, L. A., \& Saxton, J. (2008). Cognitive status and physical function in older African Americans. Journal of the American Geriatrics Society, 56, 2014-2019.

Park, H. L., O’Connell J. E., \& Thomson, R. G. (2003). A systematic review of cognitive decline in the general elderly population. International Journal of Geriatric Psychiatry, 18, 1121-1134.

Poynter, L., Kwan, J., Sayer, A. A., \& Vasallo, M. (2011). Does cognitive impairment affect rehabilitation outcome? Journal of the American Geriatrics Society, 59, 2108-2111.

Rolland, Y., Abellan van Kang, G., Nourhasemi, F., Andrieu, S., Cantet, C., Guyonnet-Gillette S., \& Vellas, B. (2009). An abnormal "one-leg balance" test predicts cognitive decline during Alzheimer's disease. Journal of Alzheimer's Disease, 16, 525-531.

Smith, P. J., Blumenthal, J. A., Hoffman, B. M., Cooper, H., Strauman, T. A., Welsh-Bohmer, K., ... Sherwood, A. (2010). Aerobic exercise and neurocognitive performance: A meta-analytic review of randomized controlled trials. Psychosomatic Medicine, 72, 239-252.

Steffen, T. M., Hacker, T. A., \& Mollinger, L. (2002). Age and gender-related test performance in community-dwelling elderly people: Six-Minute Walk Test, Berg Balance Scale, Timed Up \& Go test, and gait speeds. Physical Therapy, 82, 128-137.

van Gelder, B. M., Tijhuis, M. A., Kalmijn, S., Giampaoli, S., \& Kromhout, D. (2007). Decline in cognitive functioning is associated with a higher mortality risk. Neuroepidemiology, 28, 93-100.

Voos, M. C., Custódio, E. B., \& Malaguias, J. (2011). Relationship of executive function and educational status with functional balance in older adults. Journal of Geriatric Physical Therapy, 34, 11-18.

World Health Organization. (2011). 10 facts on ageing and the life course. Retrieved from http://www.who.int/ features/factfiles/ageing/ 